\title{
Epicardial coronary spasm due to endothelial dysfunction after spontaneous coronary artery dissection
}

\author{
V. E. Stegehuis · R. M. Dennert - T. P. van de Hoef - J. J. Piek
}

Published online: 27 November 2019

(C) The Author(s) 2019

A 70-year-old woman underwent acetylcholine provocation testing for coronary spasm, due to typical angina symptoms and absence of obstructive coronary artery disease (CAD) as revealed by repeat coronary angiography (CAG). She had a previous conservatively treated spontaneous coronary artery dissection (SCAD).

SCAD is considered a rare non-atherosclerotic cause of acute coronary syndrome, treated predominantly conservatively, as revascularisation is associated with complications. The prevalence of SCAD is higher in women (88.5\% in a recent study [1]). Moreover, women have a higher risk of symptoms and signs of myocardial ischaemia than men, but simultaneously have a $30-50 \%$ chance of having nonobstructive coronary artery disease (NOCAD) when undergoing CAG. Both SCAD and NOCAD are underdiagnosed, but awareness of these conditions is nowadays increasing [2]. The long-term effects of SCAD are largely unknown.

In the present case, coronary spasm occurred where previously a SCAD was documented (see Fig. 1). This illustrates that SCAD may cause endothelial dys-

Electronic supplementary material The online version of this article (https://doi.org/10.1007/s12471-019-01354-4) contains supplementary material, which is available to authorized users.

V. E. Stegehuis · R. M. Dennert · T. P. van de Hoef ·

J. J. Piek $(\square)$

Heart Centre, Department of Clinical and Experimental

Cardiology, Amsterdam Cardiovascular Sciences,

Amsterdam UMC, University of Amsterdam, Amsterdam,

The Netherlands

j.j.piek@amsterdamumc.nl

\section{R. M. Dennert}

Department of Cardiology, Dr. Horacio E. Oduber Hospital,

Oranjestad, Aruba function, resulting in angina symptoms. SCAD and NOCAD are characterised by a distinct risk profile, which is significantly different than that of conventional CAD [3, 4]. Vigilance for endothelial dysfunction causing angina symptoms is warranted.

Conflict of interest V.E. Stegehuis, R.M. Dennert, T.P. van de Hoef and J.J. Piek declare that they have no competing interests.

Open Access This article is distributed under the terms of the Creative Commons Attribution 4.0 International License (http://creativecommons.org/licenses/by/4.0/), which permits unrestricted use, distribution, and reproduction in any medium, provided you give appropriate credit to the original author(s) and the source, provide a link to the Creative Commons license, and indicate if changes were made.

\section{References}

1. Saw J, Starovoytov A, Humphries K, et al. Canadian spontaneous coronary artery dissection cohort study: in-hospital and 30-day outcomes. Eur Heart J.2019;40(15):1188-97.

2. Adlam D, Garcia-Guimaraes M, Maas A. Spontaneous coronary artery dissection: no longer a rare disease. Eur Heart J. 2019;40(15):1198-201.

3. Shaw J, Anderson T. Coronary endothelial dysfunction in non-obstructive coronary artery disease: risk, pathogenesis, diagnosis and therapy. Vasc Med. 2016;21(2):146-55.

4. Saw J, Aymong E, Sedlak T, et al. Spontaneous coronary artery dissection: association with predisposing arteriopathies and precipitating stressors and cardiovascular outcomes. Circ Cardiovasc Interv. 2014;7(5):645-55. 


\section{Heart Beat}

Fig. 1 Angiography of the left anterior descending artery. a Initial angiogram in 2014 showing dissection of the distal left anterior descending (LAD) artery (indicated by the red circle). b Repeat angiogram in 2018. Coronary spasm of the distal LAD artery occurring mainly at the location of the healed spontaneous coronary artery dissection

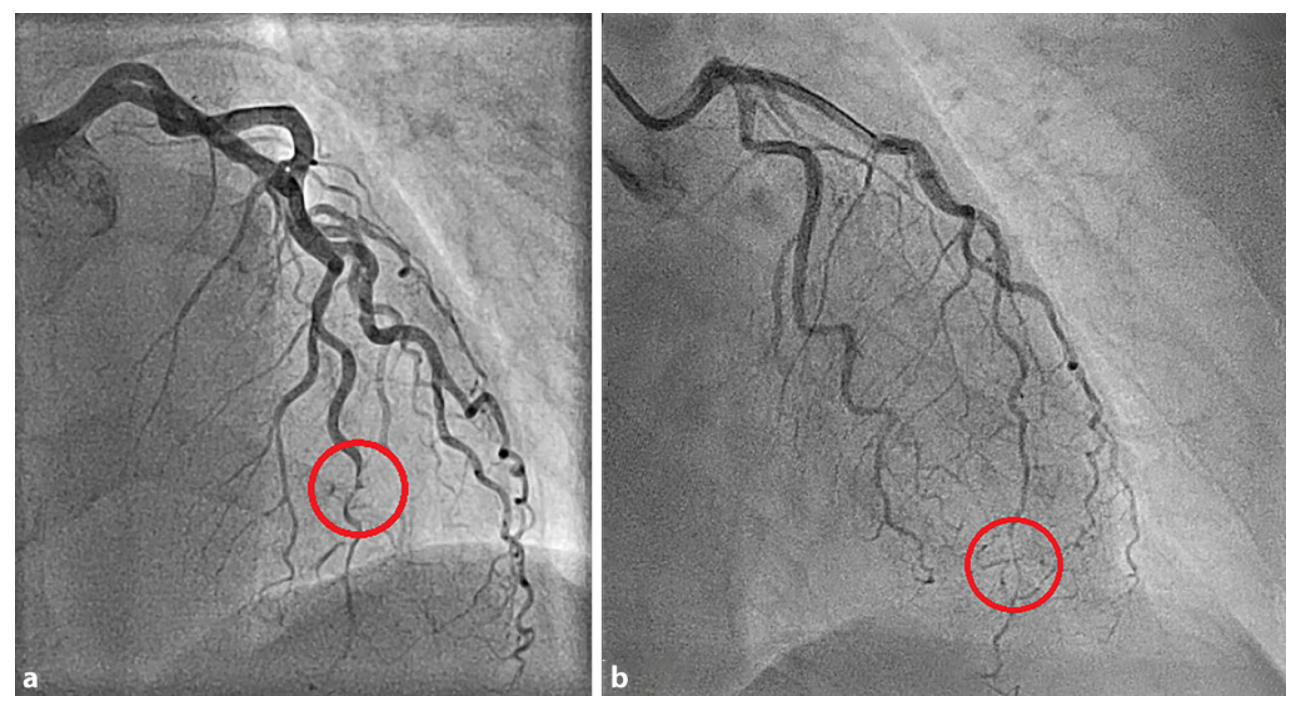

